molecules that possess energy greater than the energy of dissociation. It does not follow any longer that dissociation will be most probable when the energy of the excited entity is most nearly equal to the dissociation energy. The most probable conditions for dissociation will now be determined by the most probable jump between the two potential energy curves for the $1^{1} S$ and $1^{3} S$ levels.

This explanation of the Cario and Franck experiments does not, without further discussion, rule out the explanation that the energy goes directly into vibrational energy in the normal $1^{1} S$ level. This question of the transfer of energy from electronic to vibrational energy will be considered in a future communication.

Department of Physics, University of California, Los Angeles, California.

\section{Microseisms associated with Storms in the Indian Seas.}

THE ground is never at rest, and a seismograph provided with an aperiodic pendulum and a large magnification will always record these ever-present movements. The types are often so complicated that it is not easy to distinguish those associated with definite weather disturbances. To obviate these difficulties, a Milne-Shaw seismograph was installed some four years ago in the underground constant temperature room of the Colaba Observatory, and its working condition was so arranged that it should just cease to record microseisms when the weather was undisturbed over the neighbouring seas, as in the months of January and February, when the wind velocity seldom exceeds 20 miles per hour over the sea areas. It was then noticed that micro seisms made their appearance in the records whenever weather was disturbed over the Arabian Sea or the Bay of Bengal, so as to cause rough seas over a fairly wide area. In particular, three distinct types of microseisms were recognised, and these were associated with (1) the south-west monsoon, (2) the storms in the Arabian Sea and the Bay of Bengal, and (3) local disturbances, such as pronounced land and sea breezes. Those associated with the south-west monsoon are steady vibrations, having periods varying from 4 to 10 seconds, according to the strength of the air current over the sea.

The periods and the amplitudes of these movements are easily explained theoretically if they are considered to be standing vibrations on the earth's surface, combining to form progressive waves, analogous to Rayleigh waves, produced and maintained by the sea-waves generated by the monsoon currents. The microseisms associated with storms have periods vary ing from 4 to 6 seconds and show typical irregular variations in amplitude owing to superpositions of waves of different periods arising on account of the existence of a marked difference in wind velocity in the storm and surrounding areas. They make their appearance in the seismograms as soon as a storm has formed, and disappear only after it has passed inland and ceased to affect the sea.

The types are readily distinguished, and thus throw open to the meteorologists a new method of forecasting the existence of storms. The amplitudes of microseisms are found to be a function of the distance and the intensity of the storms. For example, the microseisms developed by the storm in the Arabian Sea, which crossed the coast between Bombay and Ratnagiri on Nov. 12, 1927, had amplitudes about four times larger than those due to a storm in the Bay of
Bengal, which crossed the coast near Nellore ten days before, but the types were identical.

During the pre-monsoon and the post-monsoon periods, when the records are almost free from monsoon microseisms, the formation and the early development of a storm are easily recognised by the gradual appearance of feeble microseisms of variable amplitude, which become more and more marked as the storm is fully developed. During the four years the instrument has been in operation, several storms formed in the Arabian Sea and the Bay of Bengal, and all of them gave rise to microseisms of this kind from the time of their formation until they passed inland and ceased to disturb the sea.

The microseisms associated with a local disturbance have large periods, varying from 20 to 30 seconds, and appear to be caused by waves over the shallow sea near the coast, for such waves have periods of exactly this order. They are certainly not due to the shaking of buildings and trees by gusts of wind, for such shakings will cause vibrations, which in an ordinary building will have periods less than $0 \cdot 1$ sec. A detailed account of these investigations is now ready and will be shortly published.

The Observatory, Bombay, S. K. BANERJI. Nov. 30.

\section{Refraction of Beams of Molecules.}

IN the Stern-Gerlach experiment the deviation of a beam of molecules in a magnetic (or electric) field is comparable to the optical case of the refraction suffered by a beam of light in traversing a medium, the refractive index of which varies in a direction perpendicular to the beam; the variation of the refractive index being analogous to the force or gradient of the field. However, in optical instruments the standard method of obtaining refraction is to allow the beam to travel from a homogeneous medium of refractive index $n_{1}$ to another of refractive index $n_{2}$. The total refraction is then independent of the rate of variation of refractive index in the interface.

It is of interest to follow out the obvious analogy for a molecular beam. In the diagram, a beam of

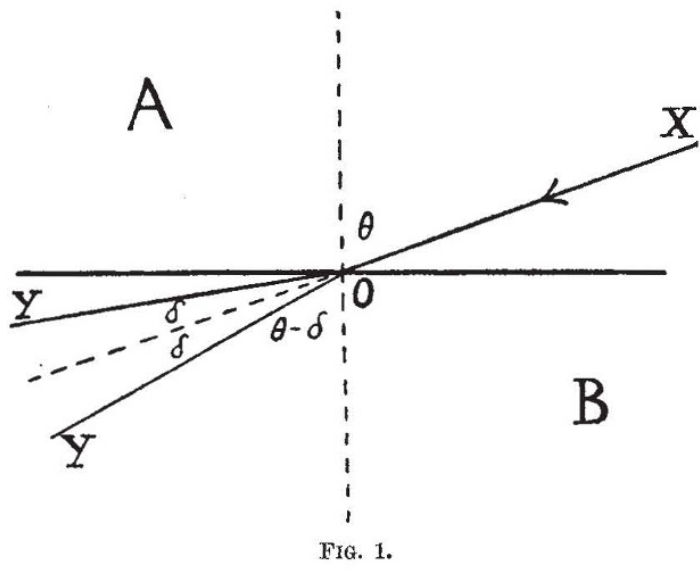

molecules $X O$ passes from a region of no magnetic field $A$ to another region $B$ in which obtains a homogeneous magnetic field $H$ perpendicular to the plane of the paper. Such a field can be produced between the flat pole pieces of a magnet. Let the beam be in the plane of symmetry between the pole pieces. We consider for simplicity a beam of alkali atoms in the normal state with kinetic energy $E$. The atoms will be orientated parallel or anti-parallel to $H$. 